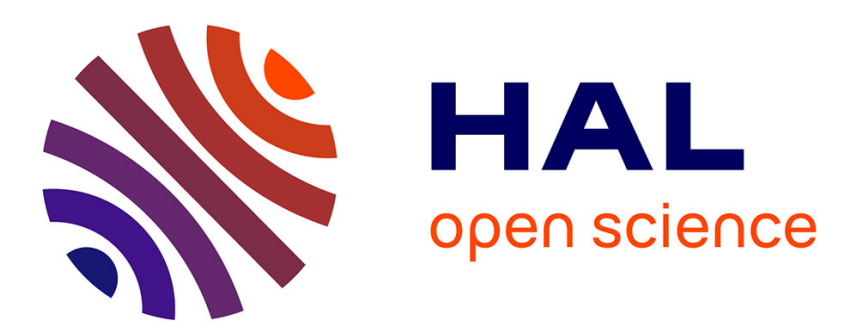

\title{
Extending the strong-field approximation of high-order harmonic generation to polar molecules: gating mechanisms and extension of the harmonic cutoff
}

\author{
Adam Etches, Lars Bojer Madsen
}

\section{- To cite this version:}

Adam Etches, Lars Bojer Madsen. Extending the strong-field approximation of high-order harmonic generation to polar molecules: gating mechanisms and extension of the harmonic cutoff. Journal of Physics B: Atomic, Molecular and Optical Physics, 2010, 43 (15), pp.155602. 10.1088/09534075/43/15/155602 . hal-00569821

\section{HAL Id: hal-00569821 \\ https://hal.science/hal-00569821}

Submitted on 25 Feb 2011

HAL is a multi-disciplinary open access archive for the deposit and dissemination of scientific research documents, whether they are published or not. The documents may come from teaching and research institutions in France or abroad, or from public or private research centers.
L'archive ouverte pluridisciplinaire $\mathbf{H A L}$, est destinée au dépôt et à la diffusion de documents scientifiques de niveau recherche, publiés ou non, émanant des établissements d'enseignement et de recherche français ou étrangers, des laboratoires publics ou privés. 


\title{
Extending the strong-field approximation of high-order harmonic generation to polar molecules: Gating mechanisms and extension of the harmonic cutoff
}

\author{
Adam Etches and Lars Bojer Madsen \\ Lundbeck Foundation Theoretical Center for Quantum System Research, \\ Department of Physics and Astronomy, Aarhus University, 8000 Aarhus C, \\ Denmark
}

\begin{abstract}
Polar molecules such as $\mathrm{CO}$ are interesting target systems for highorder harmonic generation (HHG) as they can be oriented with current laser techniques, thus allowing the study of systems without inversion symmetry. However, the asymmetry of the molecule also means that the molecular orbitals are Stark shifted in energy due to their interaction with the driving laser. We extend the strong-field approximation of HHG by incorporating the Stark shift into the Lewenstein model, and discuss its impact on two different gating mechanisms in CO. In system-induced gating an oriented target molecule serves as a gate by selecting every other half-cycle due to an increased (decreased) ionization rate. In field-induced gating the waveform of the driving laser is tailored such that the harmonic emission from an aligned molecule is damped (enhanced) every other half-cycle. We show that the Stark shift weakens the strength of system-induced gating, and also determines the relative contribution from opposite orientations in field-induced gating. Finally, we propose a novel scheme for extending the high-order harmonic cutoff by letting the two gating mechanisms counteract each other, thus allowing for a higher laser intensity without increased ionization of the target gas.
\end{abstract}

PACS numbers: 42.65.Ky, 42.65.Re

Submitted to: J. Phys. B: At. Mol. Opt. Phys. 


\section{Introduction}

High-order harmonic generation (HHG) is a highly non-linear process that takes place when an intense femtosecond laser pulse is focused onto a gas. The least bound electrons experience a force comparable to that of the Coulomb interaction with the nuclei, leading to the emission of a broad range of harmonics. HHG is often described as three separate steps. First the electron tunnels out into the continuum due to the laser interaction. The electron is then driven away from the parent ion by the laser field, and back again when the field changes its sign. Finally, the electron recombines and emits its excess kinetic energy as radiation.

Studies of HHG are largely driven by the desire to obtain a table-top source of coherent attosecond pulses reaching into the water window (284-543 eV) and above. The main problem so far has been to generate sufficiently intense attosecond pulses for practical applications. The most efficient methods depend on a single-atom signal, which is then phase matched in the target medium [1, 2]. Replacing the atomic target with a target of aligned molecules adds a degree of control, and thus offers the possibility of extending the natural obtainable harmonic cutoff.

Most models of molecular HHG are based on the simple man's model [3] or the Lewenstein model [4]. The Lewenstein model is often referred to as the strong-field approximation of HHG. Both models rely on the assumption that the initial state of the active electron is unperturbed by the electric field of the laser pulse. This assumption yields surprisingly accurate results for non-polar molecules but breaks down for polar molecules due to the dipole interaction between the molecule and the electric field.

We argue that the Lewenstein model can be extended to polar molecules by including the Stark shift on each molecular orbital. In a single-active electron model the total HHG signal is the coherent sum over the contribution from every occupied molecular orbital [5]. These orbitals will experience an energy shift in response to an electric field. As polar molecules generally have asymmetric molecular orbitals this results in a first-order Stark shift as well as higher-order shifts, which can be incorporated into the Lewenstein model by modifying the ionization potential of each orbital according to the instantaneous value of the electric field. While not describing the deformation of the target molecule, this method does allow us to study some of the features that are unique for HHG from polar molecules within a well-established framework.

As an example of the importance of including the Stark shift, we study the concept of gating in HHG. Normally, only odd harmonics are emitted due to selection rules relating to the inversion symmetry of the target and the driving laser [6, 7]. There are two ways of breaking this symmetry. One is to use an initial target, which is not symmetric along the polarization axis of the laser $[8,9,10,11,12,13]$. The other is to modify the waveform of the driving laser $[14,15,16,17,18,19,20]$.

Breaking the inversion symmetry of the target molecule is made possible, e.g., by recent advances in field-free molecular orientation [21, 22, 23, 24]. As opposed to aligned molecules, that only have a well-defined internuclear axis, oriented molecules also have a well-defined head-to-tail direction. In general, such a molecule will have a preferred direction of ionization [24, 25], which means that the harmonic contribution will be larger from one half-cycle than the adjacent. We shall refer to this gating mechanism as system-induced gating as it is the molecule itself that serves as a gate. We show that it is important to include the Stark shift in HHG from polar molecules as it tends to decrease the strength of system-induced gating. 


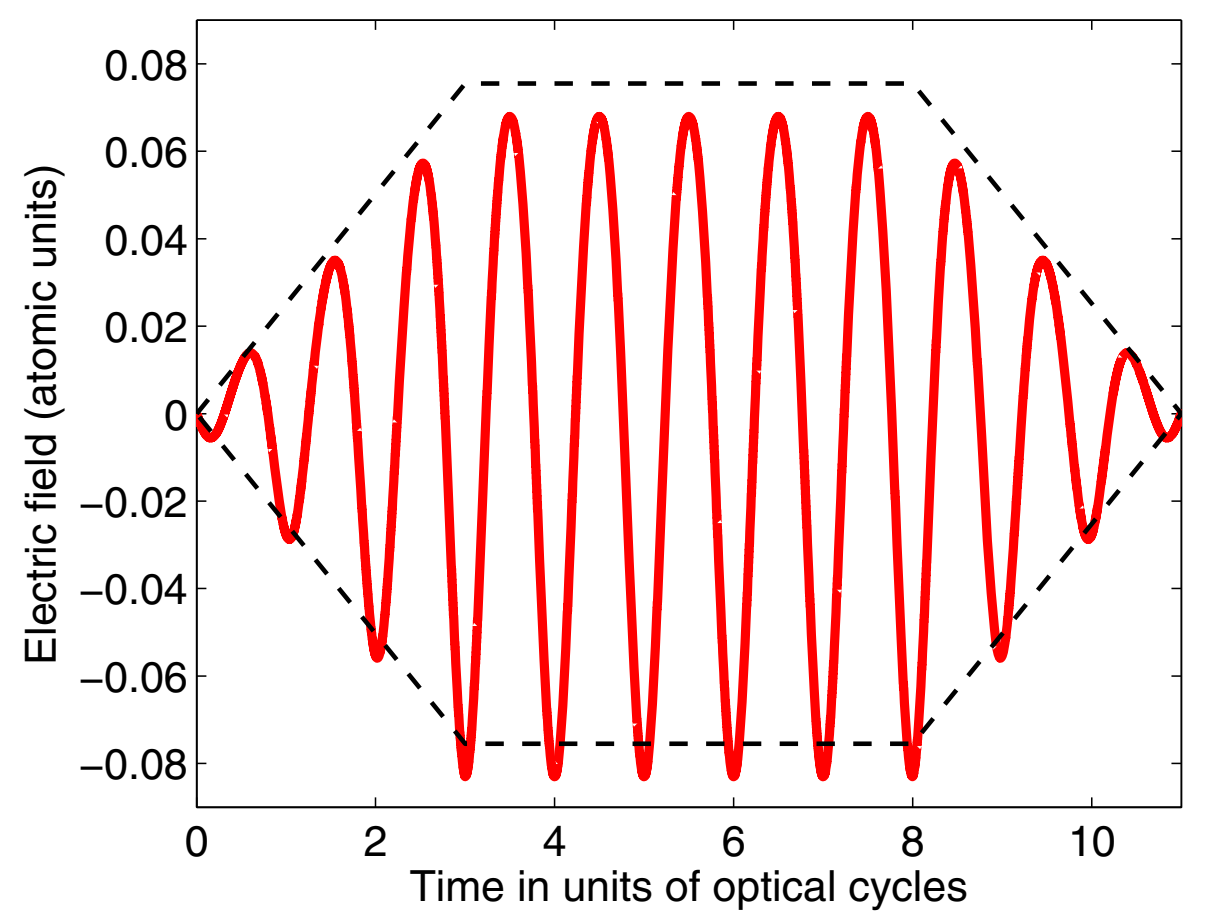

Figure 1. (Colour online) The result of adding $1 \%$ of the second harmonic in terms of intensity to an eleven cycle laser pulse with a trapezoidal envelope (indicated by the dashed line). The carrier envelope phase delay is set to zero in order to dampen (enhance) every other half-cycle. Note that the integral of the total field is zero as required of a physical pulse [26].

We also consider field-induced gating in which the waveform is changed so as to dampen the harmonic emission from an aligned target in every other half-cycle. An easy way to implement field-induced gating is to add a small amount of the second harmonic of the driving laser to the driving pulse $[15,17,20]$. As illustrated in figure 1 , the second harmonic will dampen (enhance) every other half-cycle, thus damping (enhancing) the harmonic emission. We show that the Stark shift plays an important role in determining the relative HHG contribution from opposite orientations of the aligned molecule.

Finally, we also present a novel scheme for extending the harmonic cutoff by combining system-induced and field-induced gating. We use field-induced gating to counteract the system-induced gating of an oriented molecule, thus lowering the ionization rate. This allows us to increase the intensity of the driving field without ionizing the target, which in turn leads to an extension of the harmonic cutoff. An appealing feature of our method is that it can be combined with any other cutoffextension scheme that relies on modified waveforms.

This paper is organized as follows. Our extension of the Lewenstein model is presented in section 2, supplemented by a discussion of the underlying physics behind system-induced and field-induced gating. Section 3 contains numerical results on CO, 
and section 4 sums up our conclusions. Atomic units $\left(\hbar=e=m_{e}=a_{0}=1\right)$ are used throughout unless stated otherwise.

\section{Theory}

\subsection{Lewenstein model without Stark shift}

The Lewenstein model for HHG has several variants [4, 27, 28, 29, 30, 31, 32, 33, 34, 35]. Our point of departure is the treatment in [35], where the extended stationaryphase method is used to approximate the momentum integral (describing continuum dynamics). Briefly, the HHG spectrum $S_{\mathbf{n}}(\omega)$ of the harmonic component along $\mathbf{n}$ is taken to be

$$
S_{\mathbf{n}}(\omega)=\left|\mathbf{n} \cdot \int \mathrm{e}^{\mathrm{i} \omega t} \frac{\mathrm{d}}{\mathrm{d} t}\left\langle\mathbf{v}_{\mathrm{dip}}(t)\right\rangle \mathrm{d} t\right|^{2},
$$

where $\mathbf{v}_{\text {dip }}(t)$ is the dipole velocity. The dipole velocity is split into a contribution from each molecular orbital $\lambda[5]$

$$
\left\langle\mathbf{v}_{\text {dip }}(t)\right\rangle=\sum_{\lambda} P_{\lambda}\left\langle\mathbf{v}_{\lambda}(t)\right\rangle .
$$

Introducing the Euler angles $\mathcal{R}=(\phi, \theta, \chi)$ for the molecular orientation in the laboratory frame the orientational distribution $G(\mathcal{R})$ of the molecule is factored out as a coherent sum $[5,12]$

$$
\left\langle\mathbf{v}_{\lambda}(t)\right\rangle=\int G(\mathcal{R})\left\langle\mathbf{v}_{\lambda}(\mathcal{R}, t)\right\rangle \mathrm{d} \mathcal{R} .
$$

Finally, the Lewenstein model states that given an electric field $\mathbf{F}(t)=-\partial_{t} \mathbf{A}(t)$,

$$
\begin{aligned}
\left\langle\mathbf{v}_{\lambda}(\mathcal{R}, t)\right\rangle & =\mathrm{i} \int_{0}^{t} \mathrm{~d} \tau \int \mathrm{d} \mathbf{k} \mathbf{v}_{\mathrm{rec}, \lambda}^{*}(\mathcal{R}, \mathbf{k}, t) \mathrm{e}^{-\mathrm{i} S_{\lambda}(\mathbf{k}, t, t-\tau)} \\
& \times \mathbf{F}(t-\tau) \cdot \mathbf{d}_{\text {ion }, \lambda}(\mathcal{R}, \mathbf{k}, t-\tau) \\
& + \text { c.c. },
\end{aligned}
$$

where nuclear vibration is ignored, which is justified for heavy nuclei such as carbon and oxygen. We refer to $[36,37,38,39,40,41,42]$ for a treatment that goes beyond the frozen core approximation. The momentum integral in (4) is evaluated using the extended stationary-phase method $[29,31,35]$ in order to speed up calculations. Short trajectories are selected by restricting the $\tau$-integral from zero to a $\tau_{\max }$ of two thirds of an optical cycle, which is the classical return time.

Equation (4) depends on the Fourier transform of the electronic wave function $\widetilde{\psi}_{\lambda}$ through the ionization term

$$
\mathbf{d}_{\text {ion }, \lambda}(\mathbf{k}, t-\tau)=\mathrm{i} \nabla_{\mathbf{k}+\mathbf{A}(t-\tau)} \widetilde{\psi}_{\lambda}[\mathcal{R}, \mathbf{k}+\mathbf{A}(t-\tau)],
$$

and the recombination term

$$
\mathbf{v}_{\mathrm{rec}, \lambda}(\mathbf{k}, t)=[\mathbf{k}+\mathbf{A}(t)] \widetilde{\psi}_{\lambda}[\mathcal{R}, \mathbf{k}+\mathbf{A}(t)] .
$$

Propagation in the continuum is contained in the semi-classical phase factor

$$
S_{\lambda}(\mathbf{k}, t, t-\tau)=\int_{t-\tau}^{t}\left(\frac{1}{2}\left[\mathbf{k}+\mathbf{A}\left(t^{\prime \prime}\right)\right]^{2}-E_{\lambda}\right) \mathrm{d} t^{\prime \prime},
$$

where $\left|E_{\lambda}\right|$ is the vertical ionization potential of the molecular orbital in question. 


\subsection{Lewenstein model with Stark shift}

The Lewenstein model (4) is derived by approximating the full time-dependent molecular orbital with

$$
\begin{aligned}
\left|\psi_{\lambda}(t)\right\rangle= & \mathrm{e}^{-\mathrm{i} E_{\lambda}\left(t-t_{0}\right)}\left|\psi_{\lambda}\left(t_{0}\right)\right\rangle-\mathrm{i} \int \mathrm{d} \mathbf{k} \int_{t_{0}}^{t} \mathrm{~d} t^{\prime}\left|\psi_{\mathbf{k}}(t)\right\rangle \\
& \times \mathrm{e}^{-\mathrm{i} E_{\lambda}\left(t^{\prime}-t_{0}\right)}\left\langle\psi_{\mathbf{k}}\left(t^{\prime}\right)\left|\mathbf{F}\left(t^{\prime}\right) \cdot \mathbf{r}\right| \psi_{\lambda}\left(t_{0}\right)\right\rangle
\end{aligned}
$$

Here $\left|\psi_{\lambda}\left(t_{0}\right)\right\rangle$ is the molecular orbital before the influence of the laser pulse, and $\left|\psi_{\mathbf{k}}(t)\right\rangle$ the eigenstate of a free electron in a laser field with momentum $\mathbf{k}$, a so-called Volkov state. Equation (4) is obtained by calculating the expectation value of the dipole velocity, and keeping only cross terms.

The phase factors in (8) containing $E_{\lambda}$ are the result of applying the field-free time-evolution operator on a field-free energy eigenstate

$$
\exp \left(-\mathrm{i} \int_{t_{0}}^{t} \hat{H}_{0} \mathrm{~d} t^{\prime}\right)\left|\psi_{\lambda}\left(t_{0}\right)\right\rangle=\exp \left(-\mathrm{i} E_{\lambda}\left(t-t_{0}\right)\right)\left|\psi_{\lambda}\left(t_{0}\right)\right\rangle,
$$

assuming that the effect of the laser field on the initial state can be completely ignored up until the ionization step. However, a field-free eigenstate will experience a Stark shift in response to an electric field F. Expanding in powers of the field, the shifted energy reads

$$
E_{\lambda}=E_{\lambda}^{(0)}-\mu_{\lambda}^{i} F_{i}-\frac{1}{2} \alpha_{\lambda}^{i j} F_{i} F_{j}-\frac{1}{3 !} \beta_{\lambda}^{i j k} F_{i} F_{j} F_{k}-\ldots
$$

where $\mu_{\lambda}$ is the dipole, $\alpha_{\lambda}$ the polarizability and $\beta_{\lambda}$ the first hyperpolarizability of the molecular orbital expressed in the laboratory frame. We use Einstein summation for the tensor products. The relevant molecular orbital is typically the highest occupied molecular orbital (HOMO) due to its lower ionization potential.

We propose to extend the Lewenstein model by Stark shifting the molecular orbitals according to the instantaneous value of the electric field

$$
E_{\lambda}(t)=E_{\lambda}^{(0)}-\mu_{\lambda}^{i} F_{i}(t)-\frac{1}{2} \alpha_{\lambda}^{i j} F_{i}(t) F_{j}(t)-\ldots
$$

In this quasi-static approximation it then follows that

$$
\exp \left(-\mathrm{i} E_{\lambda}\left(t-t_{0}\right)\right)\left|\psi_{\lambda}\left(t_{0}\right)\right\rangle \rightarrow \exp \left(-\mathrm{i} \int_{t_{0}}^{t} E_{\lambda}\left(t^{\prime}\right) \mathrm{d} t^{\prime}\right)\left|\psi_{\lambda}\left(t_{0}\right)\right\rangle
$$

which reduces to first-order perturbation theory if the field is only included to first order in (11). The appealing feature of our approach is that it leaves the Lewenstein model in section 2.1 unchanged except for $E_{\lambda}$ in (7) being replaced by $E_{\lambda}(t)$ in (11). The effect is thus to introduce a time-dependent ionization potential. The dipole and polarizabilities are found by calculating the energy of each molecular orbital for a range of static field strengths using standard quantum chemistry software [43], and fitting the result to a polynomial.

Our model is expected to be valid in the regime where the higher-order Stark shifts are small. In HHG experiments typical field intensities are of the order of $1-2 \times 10^{14} \mathrm{~W} / \mathrm{cm}^{2}$, corresponding to a maximal field-strength of $F_{0} \approx$ $\sqrt{2 \times 10^{14} / 3.51 \times 10^{16}}$ a.u. $=0.075$ a.u. Taking CO as an example, our quantum chemistry calculations [43] show that the HOMO, sketched in figure 2, has a permanent dipole of 1.1287 a.u. leading to a Stark shift of $17 \%$ of the ionization potential. The 


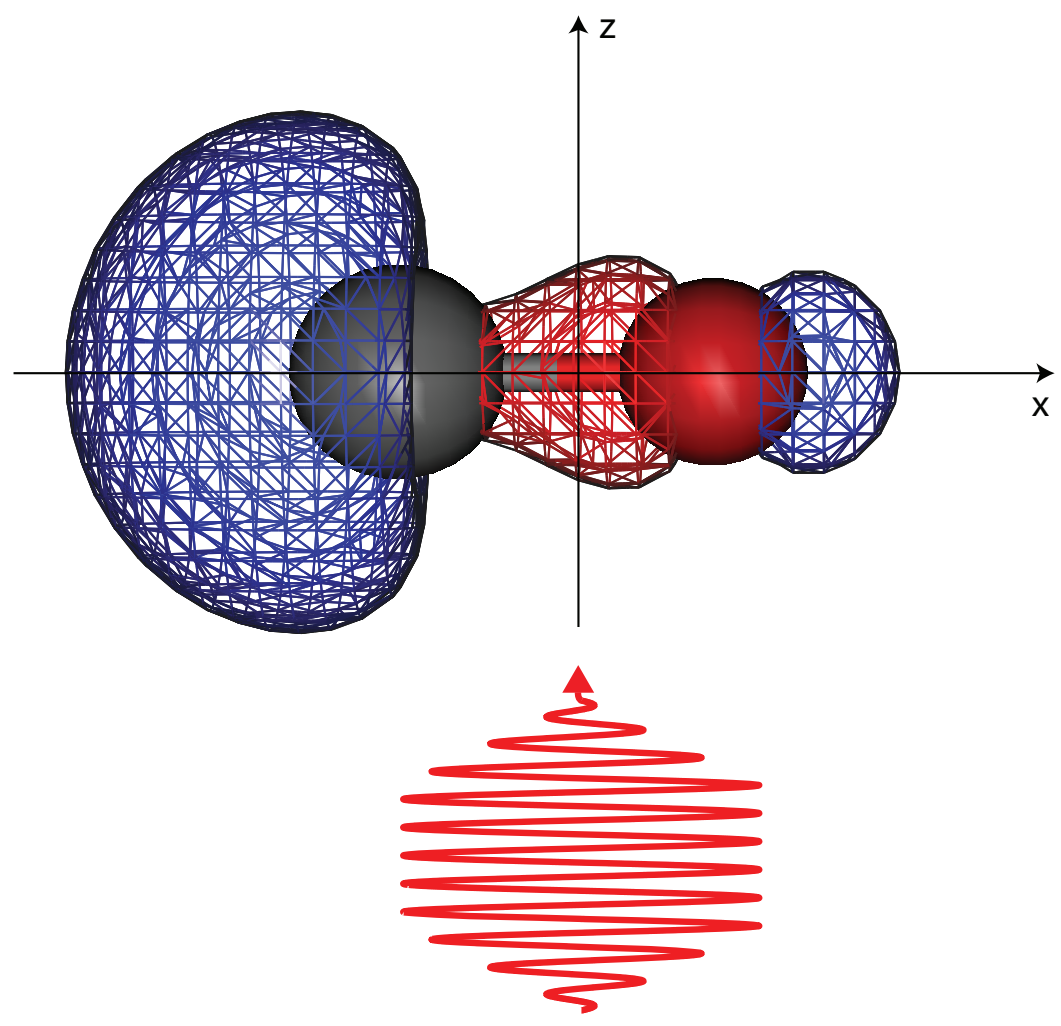

Figure 2. (Colour online) A sketch of the geometry. The molecule is oriented along the $x$ axis, which is also the polarization axis of the linearly polarized driving pulse. Shown is the highest occupied molecular orbital (HOMO) of CO obtained using standard quantum chemistry software [43]. There is a larger electron density near the carbon nucleus to the left, leading to a dipole of $1.1287 \mathrm{a}$ a. . pointing from the carbon to the oxygen nucleus. Note that the total dipole of the $\mathrm{CO}$ molecule is $0.112 \mathrm{D}=0.0441$ a.u. pointing from the oxygen to the carbon nucleus.

maximal Stark shift is shown in figure 3 for the two possible orientations. The secondorder Stark shift is much smaller than the first-order shift indicating that the field does not induce a large deformation of the molecular orbital or, equivalently, that there is not a large degree of state mixing of the bound states.

Simple considerations show why it has not previously been necessary to include the Stark shift in HHG modelling. The term that is linear in the field will always 


\section{(a) Parallel geometry}
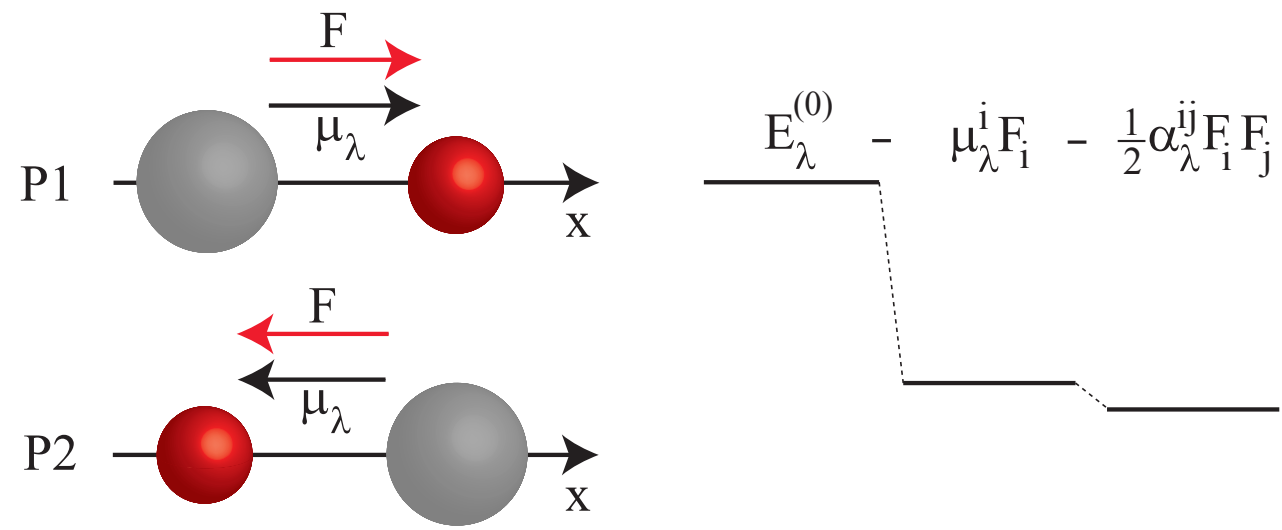

(b) Antiparallel geometry
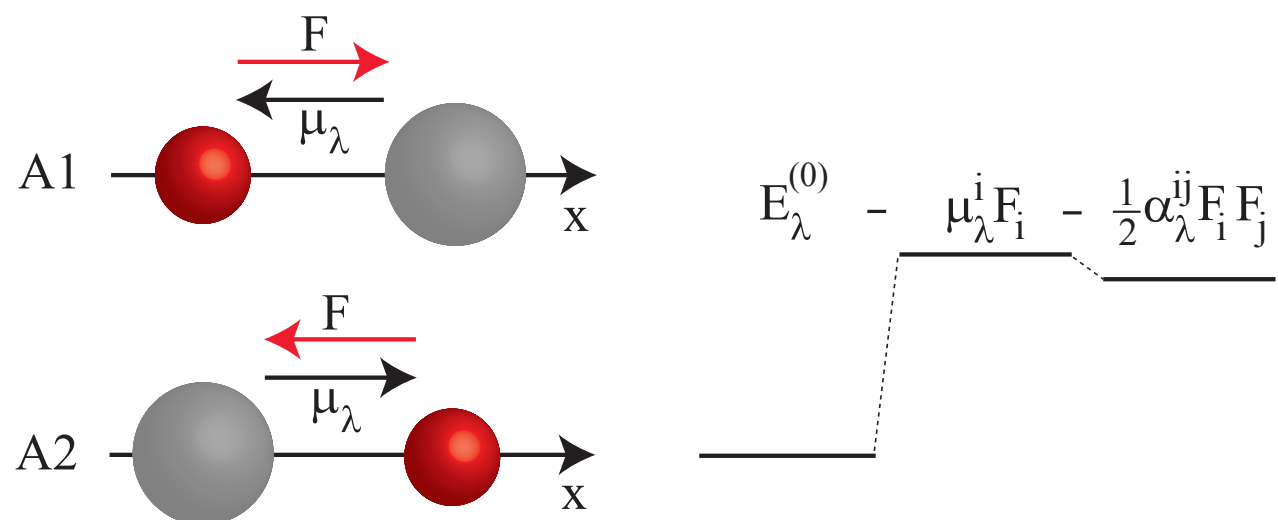

Figure 3. (Colour online) The two possible orientations of $\mathrm{CO}$ with the associated Stark shifts drawn to scale using the maximal field strength and the molecular parameters from section 3. The nuclei are drawn with an unequal size (C being the larger) to reflect the charge distribution of the HOMO in figure 2 . (a) The HOMO dipole $\mu_{\lambda}$ is parallel to the electric field $F$ so the energy of the HOMO $E_{\lambda}$ is shifted down. (b) The antiparallel geometry, in which the HOMO is shifted up in energy. The second-order induced-dipole shift caused by the polarizability $\alpha_{\lambda}$ is independent of the orientation, and is much smaller than the first-order shift. 
be exactly zero for non-polar molecules as their molecular orbitals do not possess a permanent dipole. The second-order term is symmetric with respect to the sign of the field and thus does not distinguish between half-cycles, and higher-order terms are heavily suppressed due to the typical size of $F_{0}$ in HHG studies. The net effect is a slight lowering of the energy at the peak of the cycle due to the second-order Stark shift, leading to a negligible suppression of the total HHG signal. Including the first-order Stark shift is important for polar target molecules, however. We will highlight the role that the Stark shift plays in the context of gating mechanisms.

\subsection{System-induced gating}

The first gating mechanism to be discussed is system-induced gating, where the symmetry between half-cycles in HHG is broken due to a preferred direction of ionization in the oriented target molecule [24, 25]. One possible cause of asymmetry is the asymmetric charge distribution of the molecular orbital, which follows directly from the Lewenstein model. To simplify the argument we only consider the parallel geometry in figure 2, neglect the effect of the Stark shift, and restrict ourselves to a driving field that is a sine with a constant amplitude. This means that all the following vector equations can be restricted to just the $x$ component along the polarization axis.

Applying the conventional stationary-phase approximation to the momentum integral in (4) leads to a stationary saddle-point momentum $\mathbf{k}_{\mathrm{s}}$ of [35]

$$
\mathbf{k}_{\mathrm{S}}(t, \tau)=-\frac{1}{\tau} \int_{t-\tau}^{t} \mathbf{A}\left(t^{\prime}\right) \mathrm{d} t^{\prime} .
$$

Changing half-cycle is the same as changing the sign of $\mathbf{A}$, and thus of $\mathbf{k}_{s}$, because of the sinusoidal driving pulse. The overall effect of changing half-cycle can now be deduced from (4)-(7). Using general properties of the Fourier transform

$$
\widetilde{\psi}_{\lambda}[\mathcal{R},-\mathbf{k}]=\widetilde{\psi}_{\lambda}^{*}[\mathcal{R}, \mathbf{k}]
$$

and

$$
\nabla_{-\mathbf{k}} \widetilde{\psi}_{\lambda}[\mathcal{R},-\mathbf{k}]=-\nabla_{\mathbf{k}} \widetilde{\psi}_{\lambda}^{*}[\mathcal{R}, \mathbf{k}]
$$

Changing half-cycle thus results in

$$
\mathbf{v}_{\text {rec }, \lambda}^{*}(\mathcal{R}, \mathbf{k}, t) \rightarrow-\mathbf{v}_{\text {rec }, \lambda}(\mathcal{R}, \mathbf{k}, t),
$$

and

$$
\mathbf{F}(t-\tau) \cdot \mathbf{d}_{\text {ion }, \lambda}(\mathcal{R}, \mathbf{k}, t-\tau) \rightarrow-\mathbf{F}(t-\tau) \cdot \mathbf{d}_{\text {ion }, \lambda}^{*}(\mathcal{R}, \mathbf{k}, t-\tau) .
$$

The semi-classical phase in (7) does not change so the integrand in (4) is changed from

$$
\mathrm{iv}_{\text {rec }, \lambda}^{*}(\mathcal{R}, \mathbf{k}, t) \mathrm{e}^{-\mathrm{i} S_{\lambda}(\mathbf{k}, t, t-\tau)} \mathbf{F}(t-\tau) \cdot \mathbf{d}_{\text {ion }, \lambda}(\mathcal{R}, \mathbf{k}, t-\tau)
$$

to

$$
\operatorname{iv}_{\text {rec }, \lambda}(\mathcal{R}, \mathbf{k}, t) \mathrm{e}^{-\mathrm{i} S_{\lambda}(\mathbf{k}, t, t-\tau)} \mathbf{F}(t-\tau) \cdot \mathbf{d}_{\text {ion }, \lambda}^{*}(\mathcal{R}, \mathbf{k}, t-\tau) .
$$

The contribution to the dipole velocity from either half-cycle is then obtained from (4) by taking the real part of (18) or (19) and integrating two thirds of an optical cycle. This will give different results as

$$
\operatorname{Re}\left[\mathrm{iv}_{\text {rec }}^{*} \mathrm{e}^{-\mathrm{i} S} \mathbf{F} \cdot \mathbf{d}_{\text {ion }}\right] \neq \operatorname{Re}\left[\mathrm{iv}_{\text {rec }} \mathrm{e}^{-\mathrm{i} S} \mathbf{F} \cdot \mathbf{d}_{\text {ion }}^{*}\right]
$$


when the molecular orbital is not symmetric along the polarization axis of the laser, which is exactly the case for asymmetric molecular orbitals. On the other hand, symmetric orbitals do yield the same dipole velocity in either half-cycle.

The above argument shows that one half-cycle will give a larger contribution to the HHG signal than the other given an asymmetric molecular orbital. The argument does not take into account the exchange harmonics [29,31,35] nor does it predict which half-cycle is the dominating one. It turns out that the harmonic signal is dominated by contributions corresponding to ionization in the half-cycle when the field points parallel to the dipole of the molecular orbital. In figure 2 this corresponds to the field pointing in the positive $x$ direction, thus pulling electronic charge in the negative $x$ direction. That the other half-cycle should be suppressed can be intuitively understood as a screening effect, where the main effect of the charge on the oxygen nucleus is to prevent tunnelling in the positive $x$ direction. These observations are confirmed by the numerical results in section 3 .

Reintroducing the Stark shift using (11) for $E_{\lambda}(t)$ in (7) results in the ionization potential being increased when the field is parallel to the dipole of the molecular orbital, and decreased when the field is antiparallel as illustrated in figure 3. Increasing (decreasing) the ionization potential will make it less (more) likely for the electron to tunnel into the continuum so the Stark shift tends to counteract the geometric effect just described. To account for the field-induced shifts becomes increasingly important for higher field strengths. We should stress, however, that the term system-induced gating refers to the total gating effect caused by the response of the target system to the driving field, including the Stark shift. In more sophisticated models the modification of the ionization and recombination steps due to the presence of excited states should also be considered. The permanent dipole of the molecular potential (the dipole of the unrelaxed cation) can also play a role in determining the ionization dynamics, although this effect seems to be small for CO [44].

\subsection{Field-induced gating}

Field-induced gating refers to any gating mechanism, which relies on a waveform that deviates from a pure sinusoidal field. One example is the use of pulses that are so short that tuning the carrier envelope phase allows for the selection of one particular half-cycle [19]. Another example is that of using a polarization gate, where only part of the pulse has a linear polarization that allows the ionized electron to recombine with the molecular ion $[16,18]$.

We will restrict ourselves to a very simple field-induced gating scheme. Adding a small fraction of the second harmonic to the driving pulse with a relative carrierenvelope phase delay of zero leads to every other peak in the resulting field being enhanced (suppressed) as illustrated in figure 1. HHG scales exponentially with the maximal field strength due to the first step being a tunnelling process, making it possible to effectively weaken the HHG contribution from every other half-cycle. The control parameter is the amount of second harmonic that is added to the fundamental field.

In field-induced gating the target system is assumed to be inversion symmetric along the polarization axis. This means that polar molecules are only aligned, not oriented. An aligned molecule is the coherent superposition of two antiparallel oriented molecules so by (3) the total HHG signal is a coherent sum of the individual signals [12]. At a given half-cycle, each orientation will experience either a parallel or an antiparallel 
electric field leading to system-induced gating as described in section 2.3. Assuming that the Stark shift is negligible, and referring to the orientations in figure 3, P1 will dominate over A1 in the positive half-cycle, and P2 will dominate over A2 in the negative half-cycle. The gating felt by each orientation is thus reversed in neighbouring half-cycles, ensuring the overall inversion symmetry of the HHG process.

The symmetry between the two orientations is broken in field-induced gating due to the broken symmetry of the electric field. Assuming that the maximal field-strength is in the negative $x$ direction, and ignoring the effect of the Stark shift, the contribution from $\mathrm{P} 1$ will still dominate $\mathrm{A} 1$, and $\mathrm{P} 2$ will dominate $\mathrm{A} 2$. However, $\mathrm{P} 2$ will dominate $\mathrm{P} 1$ due to the larger field-strength in the negative $x$ direction. In the limit where the system-induced gating is very strong, and the asymmetry of the field is very large, the only HHG contribution will be from the P2 geometry, making the HHG signal from field-induced gating indistinguishable to that from system-induced gating. The correspondence between the two gating mechanisms is only approximate, however, as the Stark shift weakens the effectiveness of system-induced gating. The exact weight from the different orientations and half-cycles on the total signal is determined by the size of the Stark shift.

\section{Numerical results}

Here we present numerical results for $\mathrm{CO}$ aligned along the polarization axis of the driving laser. The internuclear distance is set to the equilibrium distance $1.1283 \AA$. The molecular orbitals are obtained using standard quantum chemistry software [43]. The HOMO is a $3 \sigma$ orbital shown in figure 2. The vertical ionization potential is $14.014 \mathrm{eV}$. The HOMO-1 is a degenerate $1 \pi$ orbital with a nodal plane along the internuclear axis. The nodal plane means that the contribution from the HOMO-1 in the polarization direction [5] is heavily suppressed. Contributions from lower-lying orbitals are suppressed by the higher ionization potential so we only consider the contribution from the HOMO.

The dipole and polarizability of the HOMO are found by calculating the ionization potential for a range of static electric fields, and fitting the resulting curve to a second-order polynomial. This yields a dipole of 1.1287 a.u. pointing from $\mathrm{C}$ to $\mathrm{O}$ and a polarizability with $\alpha_{\|}=3.2332$ a.u., and $\alpha_{\perp}=2.7668$ a.u. Note that the HOMO dipole points opposite to and is much larger than the molecular dipole $0.112 \mathrm{D}=0.0441$ a.u. In our model the molecular dipole is only relevant if the orientational distribution due to an alignment pulse is needed.

The $800 \mathrm{~nm}$ driving pulse is taken to be linearly polarized along the $x$ axis. The trapezoidal envelope is sketched in figure 1. Three optical cycles are allowed for ramp-up, and three cycles for ramp-down, with five cycles at full field strength. The relatively long pulse ensures that carrier envelope phase effects play a minor role in our calculations. The field-strength is determined by the maximal intensity, which is set to $2.0 \times 10^{14} \mathrm{~W} / \mathrm{cm}^{2}$ as default. Two-colour fields as the one in figure 1 are made by adding the second harmonic with the same trapezoidal envelope and a relative carrier envelope phase of zero.

In section 3.1 we directly compare results obtained with and without including the Stark effect in (7) using (11). The Stark shift is included in section 3.2 and 3.3, where system-induced and field-induced gating are compared, and our cutoff extension scheme presented. 


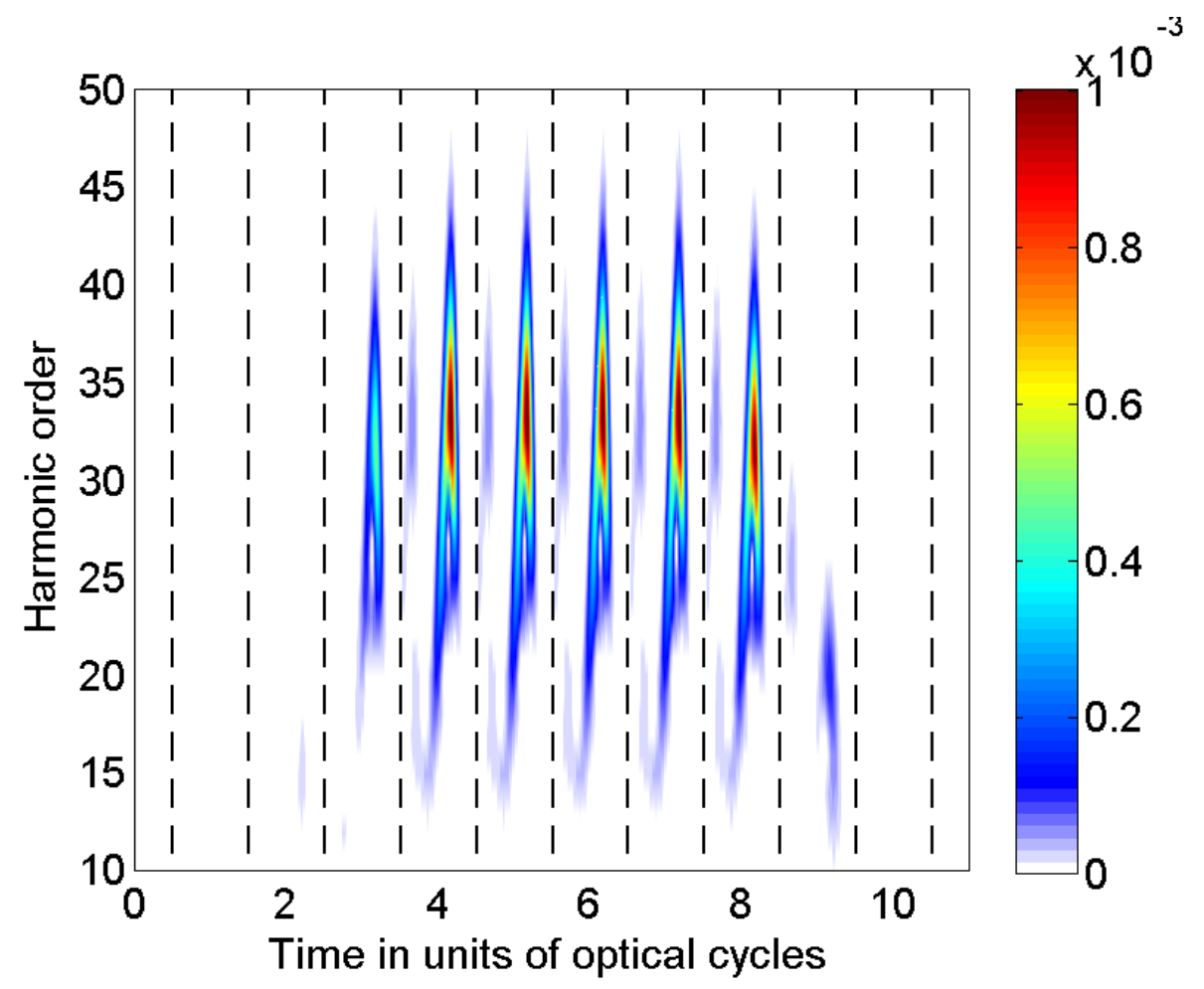

Figure 4. (Colour online) Gabor analysis illustrating system-induced gating when the Stark shift is omitted in (7). The driving field is an $800 \mathrm{~nm}$ pulse with eleven optical cycles and five optical cycles in the plateau. The peak intensity is $2.0 \times 10^{14} \mathrm{~W} / \mathrm{cm}^{2}$. The molecular target is the HOMO of CO oriented as in figure 2. The vertical dashed lines indicate the times at which the electric field peaks in the positive $x$ direction. It is clearly seen that harmonic emission is restricted to once every other half-cycle due to the asymmetry of the target.

\subsection{Importance of Stark shift}

A common way of analyzing HHG is to perform a Gabor transform $[45,46,7]$. A plot of the Gabor transform yields information on the time at which a given harmonic is emitted. Figure 4 shows such a plot without inclusion of the Stark shift given the orientation in figure 2. The times at which the electric field peaks in the positive $x$ direction are indicated by vertical dashed lines. As seen from the figure, harmonics are predominantly emitted every other half-cycle, corresponding to ionization in the positive half-cycle, and recombination roughly two thirds of an optical cycle later. Due to the asymmetry of the HOMO, the molecule itself acts as a gate for the HHG process. This is in excellent agreement with the description of system-induced gating in section 2.3 .

The geometric effect described in section 2.3 always favours the parallel geometry in figure 3. The Stark effect, however, increases the effective ionization potential in the parallel geometry, resulting in a weakening of the system-induced gating. Figure 5 shows the Gabor analysis when the Stark shift is included in (7) using (11). It is immediately clear that half-cycles suppressed in figure 4 now contribute much more 


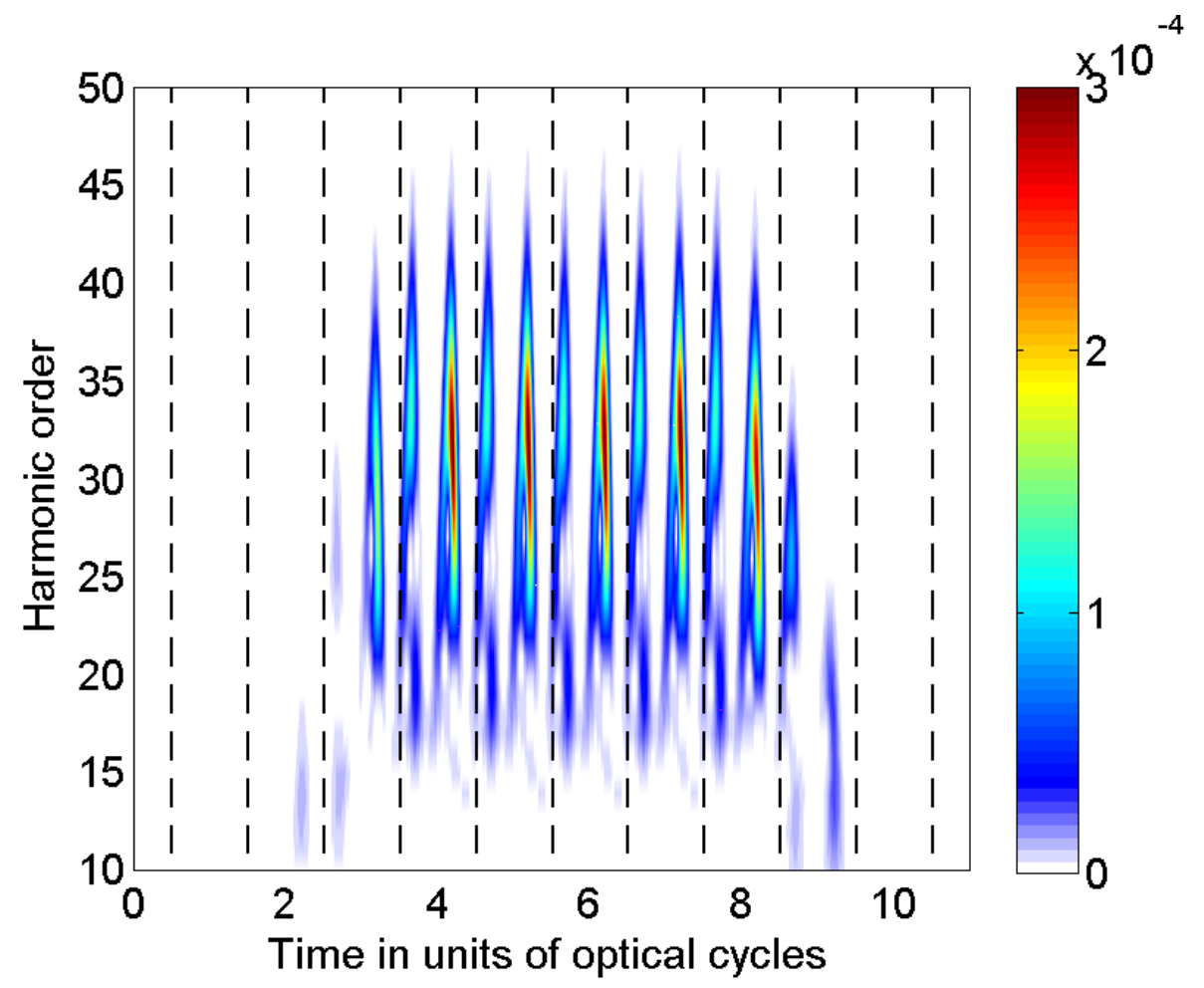

Figure 5. (Colour online) Gabor analysis illustrating the weakening of systeminduced gating caused by the Stark shift. All parameters are the same as in figure 4 except that the Stark shift is now included in (7) using (11) for $E(t)$.

to the spectrum.

Spectra obtained with and without the Stark shift (not shown) exhibit differences in the detailed structure but it is difficult to make any quantitative comparison. Roughly speaking, the spectrum for oriented CO is more rugged when the Stark shift is included. This can be understood as a consequence of added interference between half-cycles.

\subsection{Comparing gating mechanisms}

The two different gating mechanisms both imply a broken symmetry, which should result in even harmonics in the HHG spectrum. Figure 6 serves as a reference spectrum in which the target $\mathrm{CO}$ molecule is aligned, and the driving field a trapezoidal pulse with eleven cycles as described in the caption. Even harmonics are suppressed due to the inversion symmetry of both target and field $[6,7]$. Figure 7 shows the HHG spectrum when CO is oriented, thus causing system-induced gating. The even harmonics are due to the broken inversion symmetry of the target. Figure 8 shows the spectrum from aligned CO with field-induced gating implemented using $1 \%$ of the second harmonic as in figure 1. The even harmonics are due to the broken inversion symmetry of the field.

Figures 7 and 8 both contain even harmonics but differ in their detailed structure. 


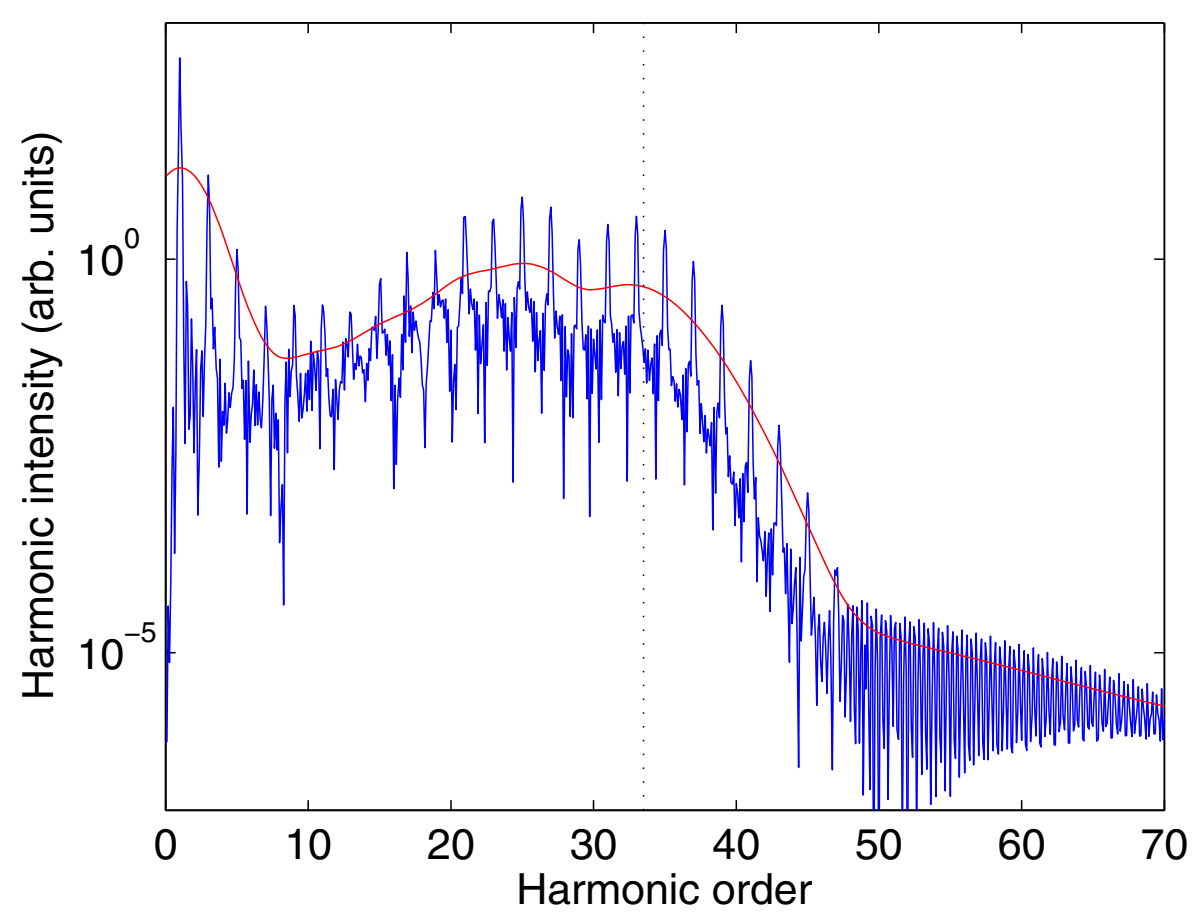

Figure 6. (Colour online) Reference HHG spectrum from aligned CO calculated with the Stark shift included in (7) as dictated by (11). The electric field is an $800 \mathrm{~nm}$ pulse with eleven optical cycles and a trapezoidal envelope with five optical cycles in the plateau. The peak intensity is $2.0 \times 10^{14} \mathrm{~W} / \mathrm{cm}^{2}$. Only odd harmonics appear due to the inversion symmetry of both target and field. The smooth (red) curve is obtained by averaging over neighbouring peaks. The vertical broken line indicates the harmonic cutoff calculated using $\omega_{\max }=I_{\mathrm{p}}+3.17 U_{\mathrm{p}}$. The Stark effect is not included in estimating the cutoff.

Figure 9 shows a Gabor analysis of the two orientations in the field-induced gating case. One orientation, figure 9(a), is seen to have its system-induced gating counteracted by the field-induced gating, and the other orientation, figure 9(b), to have its systeminduced gating enhanced. In the limit of perfect system-induced gating only the latter orientation would contribute to the spectrum, as described in section 2.4. The exact weight on each orientation is determined by the effectiveness of the system-induced gating, which depends on the Stark shift as discussed in section 3.1.

\subsection{Extending the harmonic cutoff}

We propose to extend the harmonic cutoff by suppressing the system-induced gating of an oriented polar molecule using field-induced gating as in figure 9(a). The combined effect is to suppress tunnelling into the continuum in both half-cycles, thus suppressing both HHG and ionization. Ionization of the target medium is one of the main restrictions in attaining high cutoffs as it causes depletion of the target medium as well as defocusing of the laser pulse due to free electrons. As a proof-of-principle calculation 


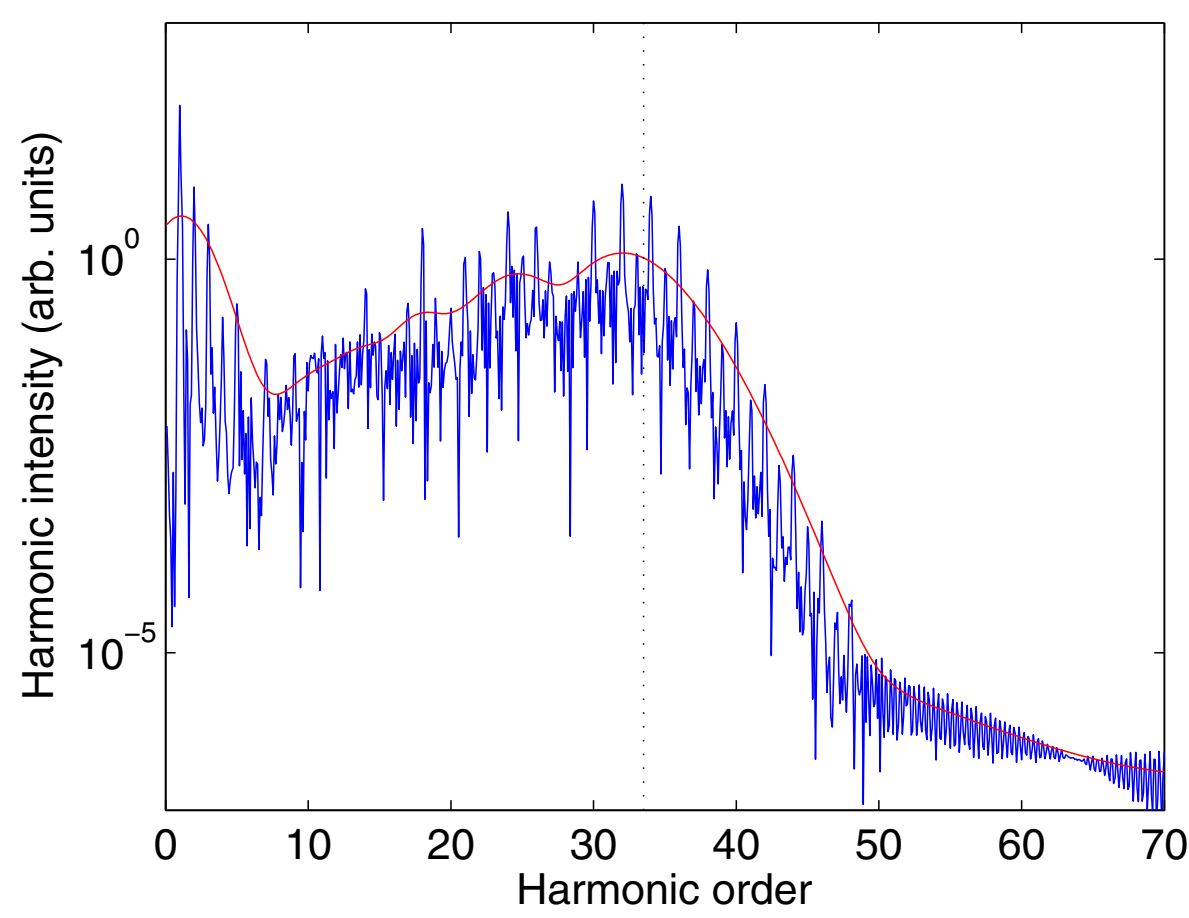

Figure 7. (Colour online) HHG spectrum using system-induced gating. All parameters are the same as in figure 6 except that the $\mathrm{CO}$ molecule is now oriented, making even harmonics possible due to the broken inversion symmetry of the target. The vertical broken line indicates the harmonic cutoff calculated using $\omega_{\max }=I_{\mathrm{p}}+3.17 U_{\mathrm{p}}$. The Stark effect is not included in estimating the cutoff.

we have used MO-ADK theory $[47,48]$ to calculate the ionization rates for oriented $\mathrm{CO}$ with and without the added second harmonic, confirming a suppressed ionization rate. According to the MO-ADK calculations an electric field with a peak intensity of $2.4 \times 10^{14} \mathrm{~W} / \mathrm{cm}^{2}$ with $1 \%$ added second harmonic causes the same amount of ionization as the original single-colour field with a peak intensity of $2.0 \times 10^{14} \mathrm{~W} / \mathrm{cm}^{2}$. The corresponding spectrum is shown in figure 10. Comparison with figure 6 shows an increase in cutoff of roughly five harmonic orders, corresponding to a $14 \%$ extension. Note that this increase can be entirely ascribed to the higher intensity of the $800 \mathrm{~nm}$ component of the driving field, as the harmonic cutoff is correctly predicted by the usual cutoff rule.

The size of the obtainable cutoff extension depends on the exact reduction of the ionization rate in a combined system-induced and field-induced gating scheme. Such a calculation is beyond the scope of this article. The scheme itself is fairly independent of the detailed dynamics, however. As long as the oriented target molecule has a preferred half-cycle, suppression of this half-cycle with a specially tailored field will allow for higher intensities, and thus higher cutoffs. This method can also be combined with driving pulses in the mid-infrared, which also lead to higher cutoff energies [49]. Finally, the argument does not depend on long pulses so short-pulse schemes [19] 


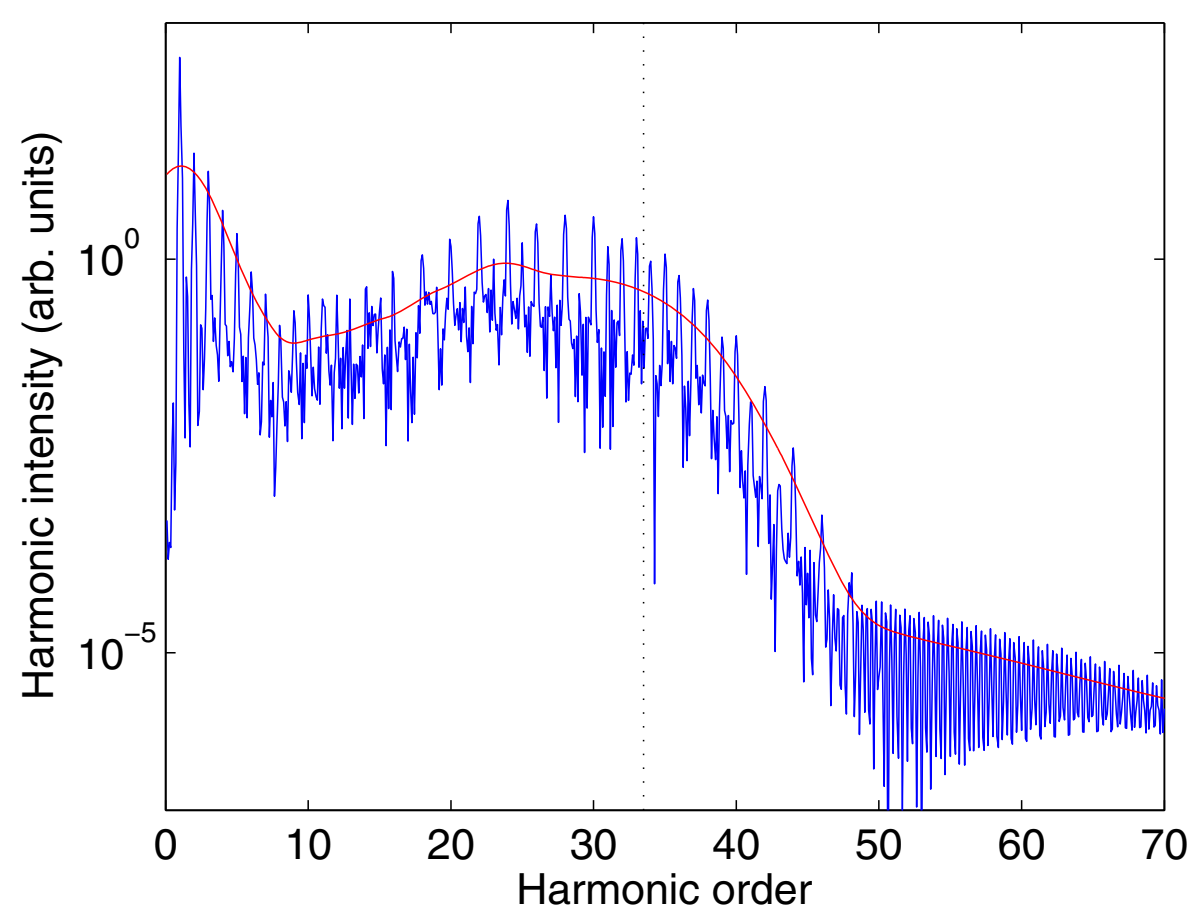

Figure 8. (Colour online) HHG spectrum using field-induced gating. The target molecule is aligned as in figure 6 but the driving field has had $1 \%$ of its second harmonic added, resulting in the field in figure 1 . The broken inversion symmetry of the field allows even harmonics to appear in the spectrum. The vertical broken line indicates the harmonic cutoff calculated using $\omega_{\max }=I_{\mathrm{p}}+3.17 U_{\mathrm{p}}$. The Stark effect and the second harmonic are not included in estimating the cutoff.

for obtaining quasi-continuous spectra will also work, as will double optical gating schemes [18].

\section{Conclusion and outlook}

We have addressed the problem of describing HHG from polar molecules in intense laser fields. The conventional strong-field approximation [4] is not expected to be valid for polar molecules due to the interaction between the permanent dipole of the fieldfree molecular orbitals and the driving field. The Lewenstein model is extended to polar molecules by applying a quasi-static approximation in which the energy levels of the molecular orbitals are Stark shifted according to the instantaneous value of the field. Qualitatively, the effect of the Stark shift is to increase (decrease) the ionization potential in half-cycles where the field points parallel (antiparallel) to the dipole of the molecular orbital thus causing a decreased (increased) contribution to the HHG signal. The second-order Stark shift causes a very slight suppression of the HHG signal for both half-cycles, and is largely negligible. Our model reduces to the standard Lewenstein model for nonpolar molecules and corresponds to first-order 
(a)
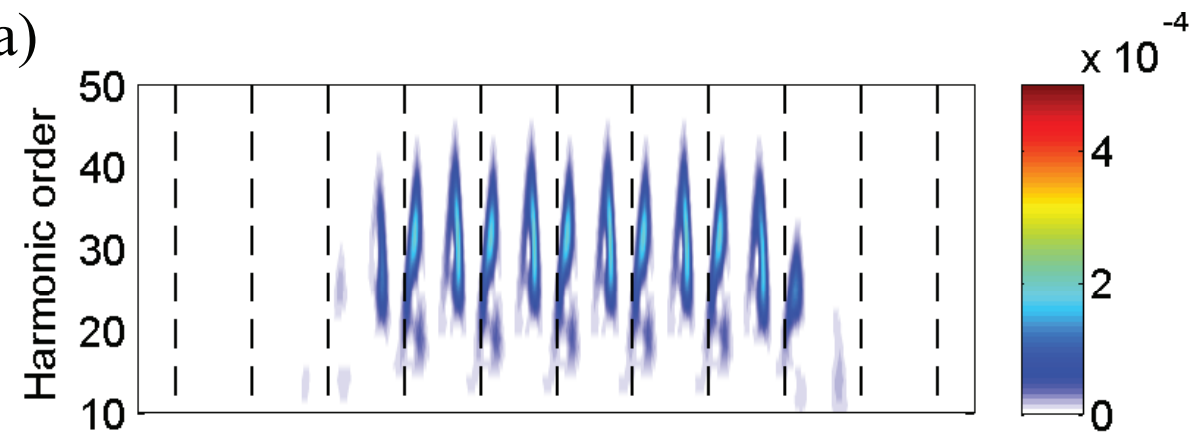

(b)

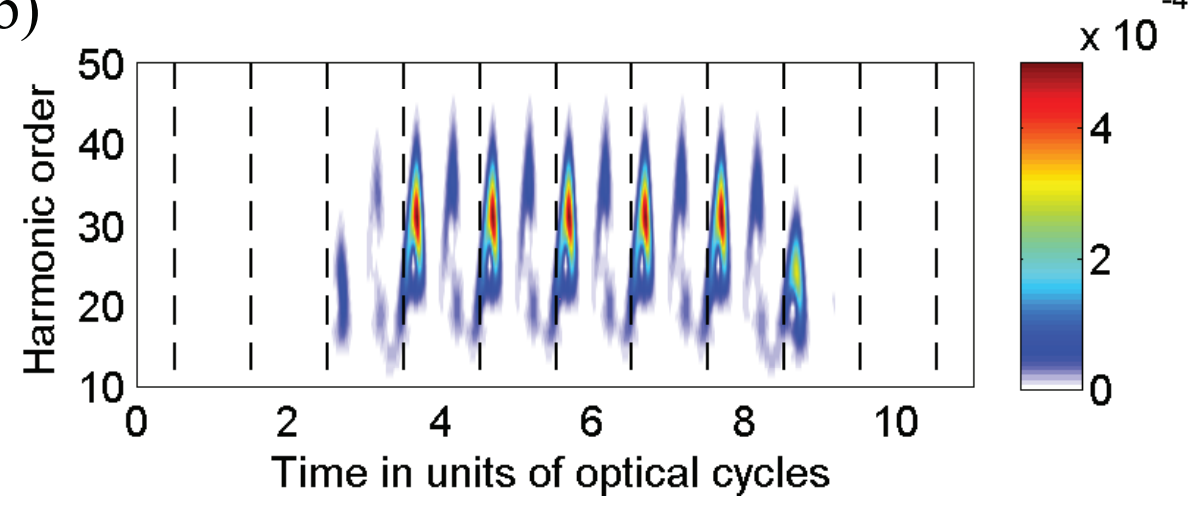

Figure 9. (Colour online) Gabor analysis illustrating the different dynamics of opposite orientations in field-induced gating. The total response of the aligned molecule is the coherent sum of the two orientations. The electric field is the same as that in figures 1 and 8. (a) The HOMO dipole is oriented in the positive $x$ direction as in figure 2. The Gabor analysis would be the one shown in figure 5 in the absence of the second harmonic so the field-induced gating clearly counteracts the system-induced gating. (b) The HOMO dipole is oriented in the negative $x$ direction causing the field-induced gating to enhance the system-induced gating.

perturbation theory in the limit of small linear Stark shifts.

The importance of including the Stark shift is evident when considering gating mechanisms. The asymmetric charge distribution from an oriented molecule leads to suppressed HHG signal in half-cycles where the field points opposite to the dipole of the molecular orbital. This leads to a geometric gating which we refer to as systeminduced gating. We show that the Stark shift suppresses the HHG signal in half-cycles where the field is parallel to the orbital dipole so the effectiveness of system-induced gating is reduced by the Stark shift. We also compare system-induced to field-induced gating, where the symmetry of the laser field is broken rather than that of the target. It is shown that even though this broken symmetry is very similar to that in systeminduced gating, the coherent nature of the HHG process means that the dynamics in a field-induced gating setup is more complicated.

Finally, we propose a novel scheme for extending the harmonic cutoff by combining system-induced and field-induced gating. By suppressing half-cycles in which an oriented molecule is most easily ionized it is possible to go to higher intensities without suffering the usual drawbacks. This method is very general and does not depend on 


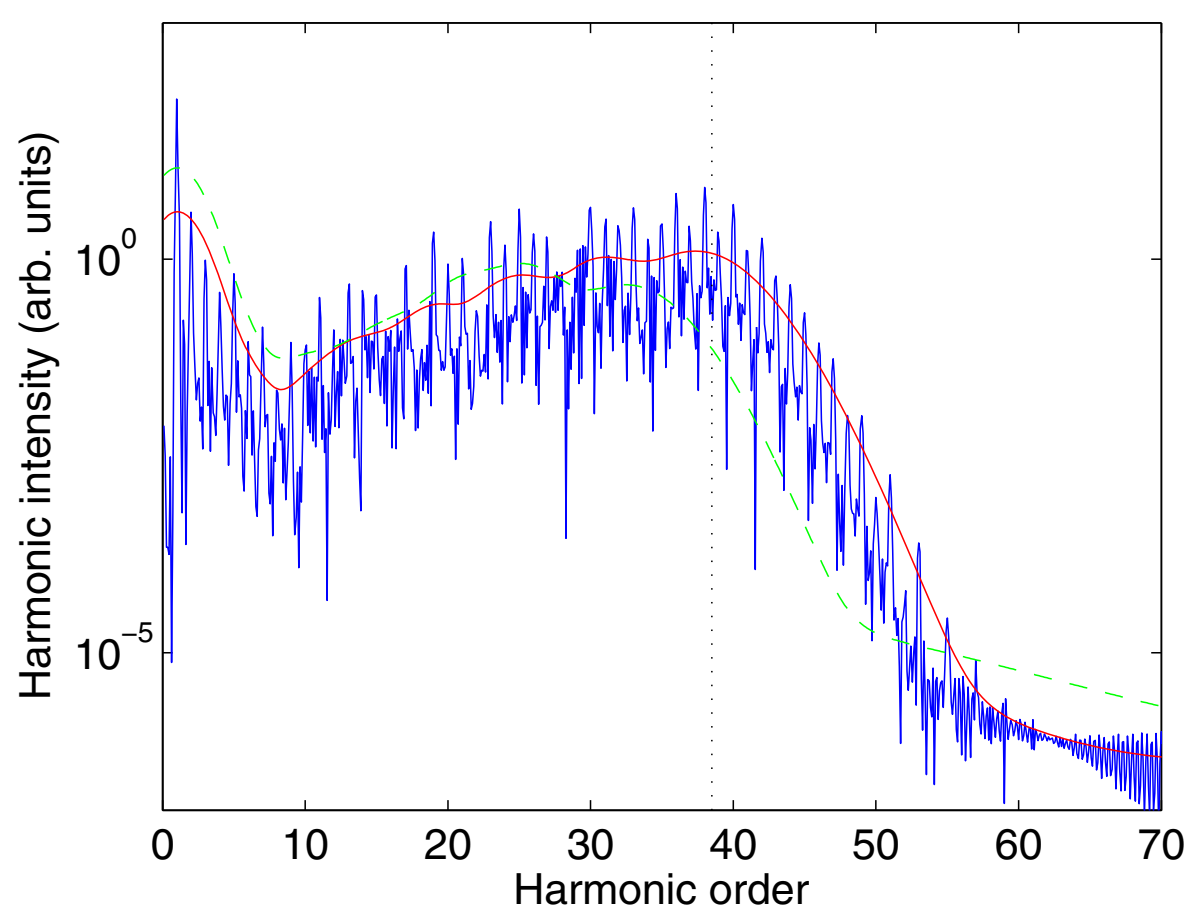

Figure 10. (Colour online) HHG spectrum illustrating the extension of the harmonic cutoff in a combined system-induced and field-induced gating scheme. The target $\mathrm{CO}$ molecule is oriented as in figure 2. The two-colour field is chosen so as to counteract the system-induced gating, resulting in a reduced ionization rate as described in the text. Using the combined gating scheme the peak intensity of the $800 \mathrm{~nm}$ component can be raised to $2.4 \times 10^{14} \mathrm{~W} / \mathrm{cm}^{2}$ before the ionization rate is the same as in figure 6 . The vertical dashed line indicates the harmonic cutoff calculated using $\omega_{\max }=I_{\mathrm{p}}+3.17 U_{\mathrm{p}}$. The Stark effect and the second harmonic are not included in estimating the cutoff. The dashed (green) line is the smoothed spectrum from figure 6 , confirming a $14 \%$ extension of the harmonic cutoff energy.

the details of our modelling. A proof-of-principle calculation indicates that increases on the order of $14 \%$ are obtainable.

In our current work we have focused on the influence that the Stark shift plays on the spectrum. There is currently much interest in gaining information about HHG from the phases of individual harmonics. Such phases are experimentally available using, e.g., the RABITT technique [50] or by performing mixed gas experiments [51]. In order to extract the influence of the Stark shift on the harmonic phases it is necessary to have a reference that is uninfluenced by the Stark shift. Krypton would be a good second species in a mixed gas experiment as it has nearly the same ionization potential as $\mathrm{CO}$ while only experiencing weak higher-order Stark shifts. 


\section{Acknowledgments}

The authors would like to thank Mahmoud Abu-Samha for calculating the dipole and polarizabilities of $\mathrm{CO}$, and Christian Bruun Madsen for helpful discussions.

\section{References}

[1] Gibson E A et al. 2003 Science 30295

[2] Takahashi E J, Kanai T, Ishikawa K L, Nabekawa Y and Midorikawa K 2008 Phys. Rev. Lett. 101253901

[3] Corkum, P B 1993 Phys. Rev. Lett. 711994

[4] Lewenstein M, Balcou Ph, Ivanov M Y, L'Huillier A and Corkum P B 1994 Phys. Rev. A 49 2117

[5] Madsen C B and Madsen L B 2007 Phys. Rev. A 76043419

[6] Alon O E, Averbukh V and Moiseyev N 1998 Phys. Rev. Lett. 803743

[7] Kamta G L and Bandrauk A D 2004 Phys. Rev. A $70011404(\mathrm{R})$

[8] Kreibich T, Lein M, Engel V and Gross E K U 2001 Phys. Rev. Lett. 87103901

[9] Kamta G L, Bandrauk A D and Corkum P B 2005 J. Phys. B: At. Mol. Opt. Phys. 38 L339

[10] Heslar J, Carrera J, Telnov D and Chu S 2007 Int. J. Quant. Chem. 1073159

[11] Lan P, Lu P, Cao W, Li Y and Wang X 2007 Phys. Rev. A 76021801

[12] Madsen C B, Mouritzen A S, Kjeldsen T K and Madsen L B 2007 Phys. Rev. A 76035401

[13] Zhang Q, Lu P, Hong W, Liao Q and Wang S 2009 Phys. Rev. A 80033405

[14] Kim I J, Kim C M, Kim H T, Lee G H, Lee Y S, Park J Y, Cho D J and Nam C H 2005 Phys. Rev. Lett. 94243901

[15] Dudovich N, Smirnova O, Levesque J, Mairesse Y, Ivanov M Y, Villeneuve D M and Corkum P B 2006 Nature Physics 2781

[16] Dudovich N, Levesque J, Smirnova O, Zeidler D, Comtois D, Ivanov M Y, Villeneuve D M and Corkum P B 2006 Phys. Rev. Lett. 97253903

[17] Pfeifer T, Gallmann L, Abel M J, Neumark D M and Leone S R 2006 Opt. Lett. 31975

[18] Mashiko H, Gilbertson S, Li C, Khan S D, Shakya M M, Moon E and Chang Z 2008 Phys. Rev. Lett. 100103906

[19] Goulielmakis E et al. 2008 Science 320, 1614

[20] Mauritsson J, Dahlström J M, Mansten E and Fordell T 2009 J. Phys. B: At. Mol. Opt. Phys. 42134003

[21] Stapelfeldt H and Seideman T 2003 Rev. Mod. Phys. 75543

[22] De, S et al. 2009 Phys. Rev. Lett. 103153002

[23] Holmegaard L, Nielsen J H, Nevo Iftach, Stapelfeldt H, Filsinger F, Küpper J and Meijer G 2009 Phys. Rev. Lett. 102023001

[24] Holmegaard L et al. 2010 Nature Physics 6428

[25] Kamta G L and Bandrauk A D 2005 Phys. Rev. Lett. 94203003

[26] Madsen L B 2002 Phys. Rev. A 65053417

[27] Salières P et al. 2001 Science 292902

[28] Zhou X X, Tong X M, Zhao Z X and Lin C D 2005 Phys. Rev. A 71 061801(R)

[29] Chirilă C C and Lein M 2006 Phys. Rev. A $\mathbf{7 3} 023410$

[30] Chirilă C C and Lein M 2009 Phys. Rev. A 80013405

[31] de Morisson Faria C Figueira 2007 Phys. Rev. A 76043407

[32] Smirnova O, Patchkovskii S, Mairesse Y, Dudovich N, Villeneuve D, Corkum P and Ivanov M Y 2009 Phys. Rev. Lett. 102063601

[33] Le A-T, Lucchese R R, Tonzani S, Morishita T and Lin C D 2009 Phys. Rev. A 80013401

[34] Odžak S and Milošević 2009 Phys. Rev. A 79023414

[35] Etches A, Madsen C B and Madsen L B 2010 Phys. Rev. A 81013409

[36] Lein M 2005 Phys. Rev. Lett. 94053004

[37] Walters Z B, Tonzani S and Greene C H 2007 J. Phys. B 40 F277

[38] Patchkovskii S 2009 Phys. Rev. Lett. 102253602

[39] Baker S, Robinson J S, Haworth C A, Teng H, Smith R A, Chirilă C C, Lein M, Tisch J W G and Marangos J P 2006 Science $\mathbf{3 1 2} 424$

[40] Baker S et al. 2008 Phys. Rev. Lett. 101053901

[41] Madsen C B and Madsen L B 2006 Phys. Rev. A 74023403

[42] Madsen C B, Abu-samha M and Madsen L B 2010 Phys. Rev. A 81043413

[43] Schmidt M W 1993 et al. J. Compt. Chem. 141347 
[44] Abu-samha M and Madsen L B In Preparation

[45] Antoine P, Piraux B and Maquet A 1995 Phys. Rev. A 51 R1750

[46] Chirilă C C, Dreissigacker I, van der Zwan E V and Lein M 2010 Phys. Rev. A 81033412

[47] Tong X M, Zhao Z X and Lin C D 2002 Phys. Rev. A 66033402

[48] Kjeldsen T K and Madsen L B 2005 Phys. Rev. A 71023411

[49] Shiner A D, Trallero-Herrero C, Kajumba N, Bandulet H-C, Comtois D, Légaré F, Giguère M, Kieffer J-C, Corkum P B and Villeneuve D M 2009 Phys. Rev. Lett 103073902

[50] Haessler S et al. 2010 Nature Physics 6200

[51] McFarland B K, Farrell J P, Bucksbaum P H and Gühr M 2009 Phys. Rev. A 80033412 